\title{
Improved Hemodynamics with the Use of Prophylactic Infusion of Epinephrine and/or Norepinephrine during Transcatheter Aortic Valve Replacement (TAVR)
}

\author{
Walter Bethune, Steven Konstadt, Giuseppe Trunfio, Lynn Belliveau, Mark Kronenfeld, \\ Charles Keilin, Dennis E. Feierman \\ Department of Anesthesiology, Maimonides Medical Center, Brooklyn, USA \\ Email: dfeierman@maimonidesmed.org \\ Received 6 May 2015; accepted 21 June 2015; published 24 June 2015 \\ Copyright (C) 2015 by authors and Scientific Research Publishing Inc. \\ This work is licensed under the Creative Commons Attribution International License (CC BY). \\ http://creativecommons.org/licenses/by/4.0/

(c) (7) Open Access

\begin{abstract}
Background: Transcatheter Aortic Valve Replacement (TAVR) is a new minimally-invasive surgical procedure in which a bioprosthetic aortic valve is placed via a small skin incision in the groin, over either the left or right iliac artery. TAVR was recently approved by the FDA as a treatment option for aortic stenosis in patients who may be too frail to undergo open heart surgery. Anesthetic management of TAVR is complicated by the fact that rapid left ventricular pacing (to at least 180 beats per minute) is required at multiple points throughout the procedure. Aim: This rapid pacing creates a profound pathophysiologic stress on the heart, sometimes leading to severe left ventricular dysfunction and resultant complete hemodynamic collapse. We report that the use of prophylactic infusion of epinephrine and/or norepinephrine in patients undergoing TAVR results in improved recovery from rapid pacing as reflected by intraoperative trends in systolic systemic blood pressure and systolic pulmonary arterial pressure. Cases: Here we report three cases. During the first of these, we reacted to intraoperative hemodynamic changes by administering boluses of vasoactive medications as needed. During the other two cases, we preemptively infused vasopressors to facilitate a more rapid recovery from some of the hemodynamic disturbance associated with either TAVR or rapid pacing. Conclusion: The two patients in this series who were managed with a preemptive strategy had higher ratios of systemic systolic blood pressure to pulmonary arterial systolic blood pressure at the end of the case than did the patient who was managed reactively. This suggests that the preemptive strategy may lead to decreased left ventricular impairment and improved overall cardiac function after TAVR.
\end{abstract}




\section{Keywords}

\section{TAVR, Hemodynamics, Aortic Valve}

\section{Introduction}

TAVR is an exciting new technology. The first TAVR was performed in 2002. The Edwards SAPIEN valve and the Medtronic CoreValve (the two most commonly used TAVR prostheses at present) were both approved for commercial use in Europe in 2007. The SAPIEN valve was FDA approved for commercial use in inoperable patients in the USA in 2011 and for high-risk US surgical candidates in 2012. This cohort of patients has a particularly grim prognosis; they can expect a 1-year survival probability of less than $50 \%$ and a 3-year survival of close to zero. The CoreValve was FDA approved for this patient population in the US only earlier this year [1].

As the procedure has only been performed widely since 2011, the currently existing literature on TAVR is sparse. In this section, we briefly outline three publications that provide background pertinent to our project. First is the landmark study that establishes the safety and efficacy of TAVR-leading ultimately to FDA approval. Second is the most complete review article to date which describes anesthetic management of TAVR. To our knowledge, a retrospective study of alternative intraoperative hemodynamic management strategies-such as we here describe- has not yet been performed. Patients undergoing TAVR typically undergo general anesthesia, including placement of a temporary subendocardial pacing lead, at least one large bore intravenous line, warming, an arterial line, and transesophageal echocardiography [1] [2]. Recently, Klein published a review on TAVR and its controversies and complications [3]. A pulmonary artery catheter is used when abnormal left ventricular function and/or other cardiac pathology is present.

During TAVR, accurate sizing and positioning of the prosthetic valve are absolutely critical: a malpositioned valve may cause sudden and life-threatening complications such as valve embolization, occlusion of the coronary ostia or acute left ventricular distention secondary to severe aortic insufficiency. 3D imaging by CT scan, aortic annulus dilation and the deployment of the new valve require significantly reduced cardiac ejection; this is achieved by rapid ventricular pacing ( 180 beats/min). Rapid ventricular pacing causes a brief but extreme reduction is systemic blood pressure and cardiac output. The immediate reestablishment of normal blood pressure and cardiac output is paramount in these patients, who have little or no physiologic reserve. Hence anesthetic management of TAVR also includes early identification and treatment of intravascular fluid volume depletion as well as the use of positive inotropic and/or vasopressor agents in patients with low cardiac output, severe left and/or right ventricular dysfunction or severe pulmonary hypertension.

During rapid ventricular pacing we expect a profound decrease in blood pressure: This is in fact our goal. Hypotension is needed to prevent an extreme increase in afterload on the left ventricle during balloon dilation, a step which is necessary in order to facilitate subsequent positioning and deployment of the prosthetic valve. Failure on the surgical team's part to appropriately decrease the blood pressure could cause the left ventricle to fail and irreversibly dilate as the left ventricular outflow tract (LVOT) is completely occluded during balloon dilation and valve deployment. We have recently changed our anesthetic management approach to these cases. Our previous approach was to treat hemodynamic derangements as needed throughout the case (reactive management). We now preemptively start infusions of vasoactive medications (typically including epinephrine and/ or norepinephrine) in order to mitigate or prevent the left ventricular dysfunction and resultant hemodynamic instability that often occurs during TAVR. We here present a representative sample of 3 cases from our early experience: two of these in which our newer (preemptive) management strategy is employed and one in which our prior (reactive) strategy is used, in order to illustrate the efficacy of our current strategy for intraoperative hemodynamic management of these critically ill patients.

\section{Methods and Results}

\subsection{Methods}

IRB approval was obtained prior to the beginning of our retrospective review. This is a presentation of 3 representative cases from our initial review. The "reactive management" case was one of the very first TAVR cases performed at our institution. The two "preemptive management" cases were two of the first that our team per- 
formed after formulating our aforementioned thesis and empirically changing our approach. As our team's experience progressed, we formed the clinical impression that administering epinephrine and/or norepinephrine more proactively, as continuous low-dose infusions started prior to rapid pacing — rather than as reactive boluses after pacing —allowed for a faster hemodynamic recovery and overall smoother intraoperative course. Our primary variables were systolic systemic pressures and pulmonary systolic pressures.

\subsection{Results}

Figure 1 and Figure 2 are based on data that included one patient managed reactively (patient 1: Reactive 1) and two patients treated proactively (patients 2 and 3: Preemptive 2 and 3). Patient 2 was started on a combination of epinephrine and norepinephrine. Patient 3 was started on epinephrine alone. Initially, our TAVR cases were managed using reactive treatment as is shown in both Figure 1 and Figure 2, patient 1: reactive 1. ART SYS pressures: before rapid pacing was $123 \mathrm{mmHg}$, and remained stable at $121 \mathrm{mmHg}$ until after balloon dilation where it dropped to $91 \mathrm{mmHg}$, as expected (the decrease in BP secondary to aortic regurgitation) and after deployment of the valve was BP returned to $143 \mathrm{mmHg}$ and remained fairly stable until the end of the case as Art sys BP was $120 \mathrm{mmHg}$. In case 2 and 3 (preemptive treatment), we see that after balloon dilatation the systolic pressure remained higher, possible secondary to the pre-emptive vaso-pressors started in this case (Figure 1, "aortic regurgitation"). In case 2 the patient was started on both epinephrine and norepinephrine; whereas in case 3 , the patient was only started on epinephrine.

Following the trend in pulmonary arterial pressures is an indirect means of assessing cardiac function. In the absence of primary pulmonary hypertension, acutely elevated pulmonary arterial pressures may indicate the new onset of cardiac dysfunction. PUL SYS pressures in case 1 showed an increase throughout the case that started to abate near the end of the case, probably reflecting LV dysfunction and/or dilatation secondary to LV dysfunction/distention the end of the case, PUL SYS pressure was still elevated compared to baseline (Figure 2, "Reactive" "Pre-surg” vs. "Late”). In cases 2 and 3, PUL SYS pressures improved: they were lower at the end of cases 2 and 3 when compared to the initial pressures and compared to case 1 (Figure 2, Preemptive 1 \& 2, "Late"): which appear to indicate that the left ventricle is functioning better.

$$
\rightarrow \text { Reactive } \quad \text { - }- \text { Preemptive } 1 \quad \rightarrow-\text { Preemptive } 2
$$

Systemic Systolic Pressures

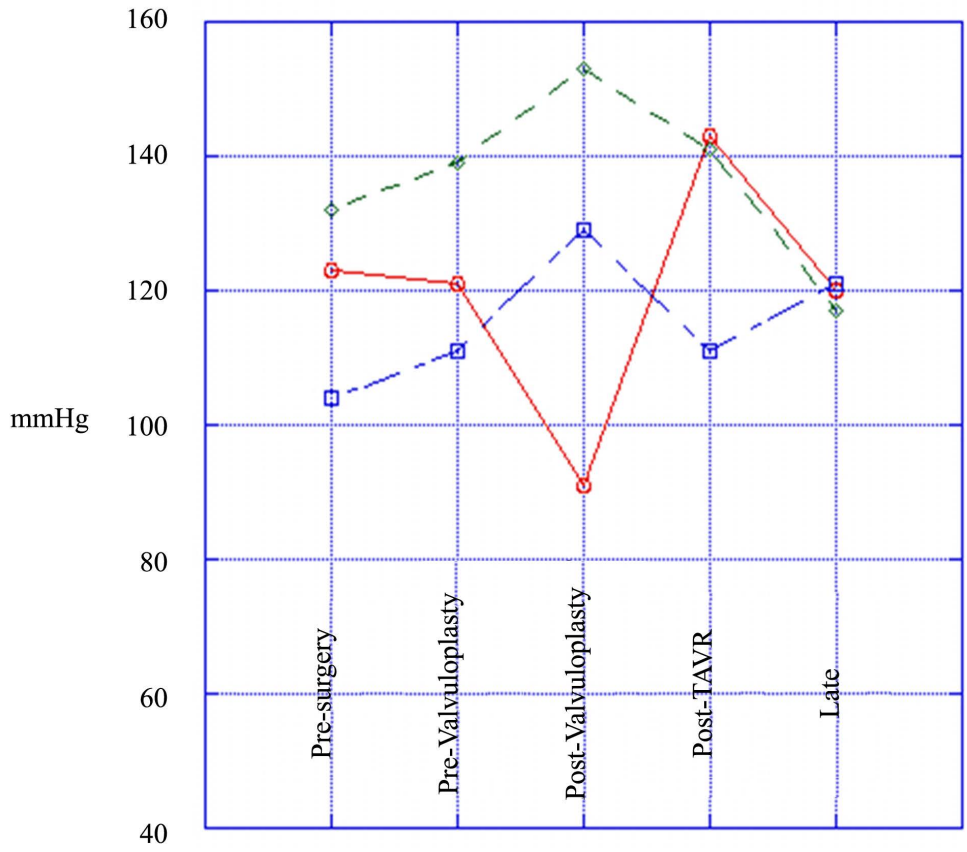

Figure 1. Changes in systemic systolic pressures at different stages of TAVR. 


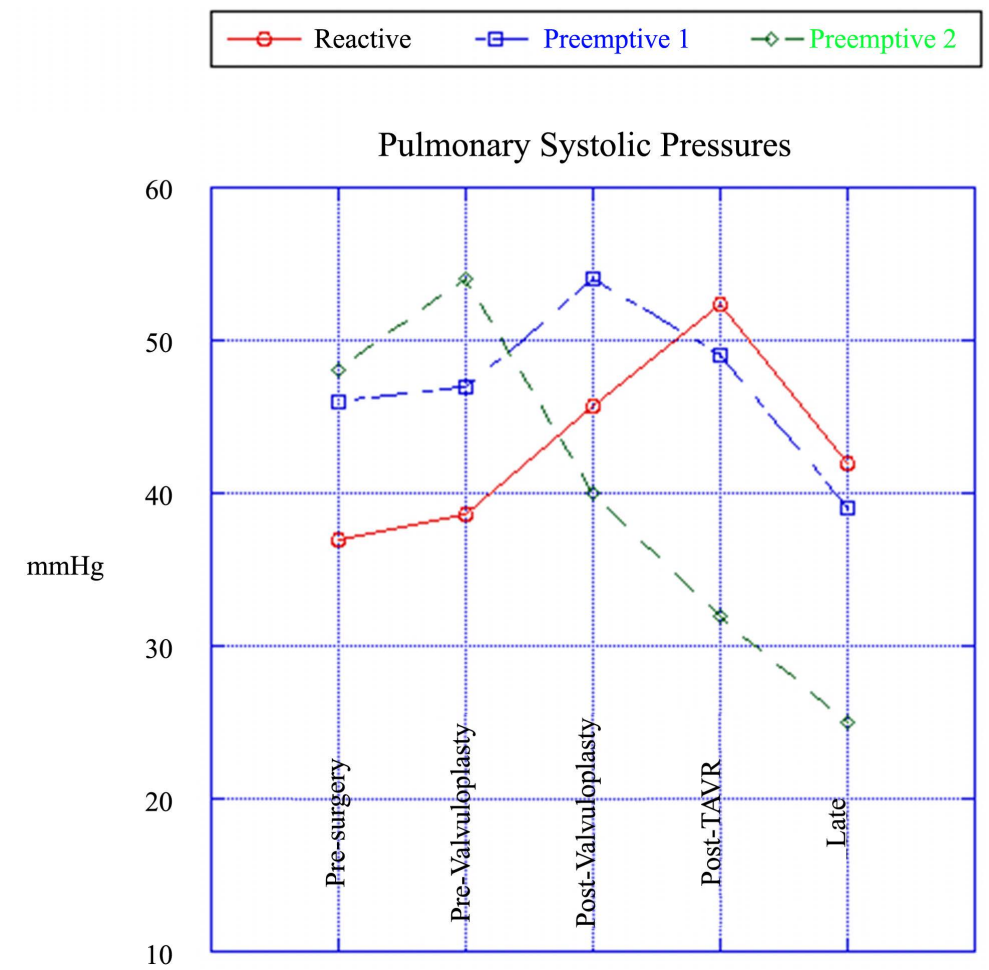

Figure 2. Changes in pulmonary systolic pressures at different stages of TAVR.

\section{Discussion}

TAVR is a relatively new technology and, as we are still at the early stages of its development, gathering data to improve safety is definitely needed. Currently, from a scientific perspective, we don't know the best approach to intraoperative hemodynamic management: we use our years of training and experience to determine empirically how to proceed. At our institution, as we here describe, we now preemptively use infusions of vasoactive drugs during TAVR. In particular, this approach seems to facilitate the heart's ability to rebound from the pathophysiologic stresses imposed by two necessary steps of the TAVR procedure; rapid ventricular pacing and balloon valvuloplasty (which often cause acute severe aortic regurgitation).

In our approach to these cases, optimal selection and dosing of preemptively administered vasoactive agents depend primarily on an assessment of the patient's baseline left ventricular function: patients with decreased LV function typically receive epinephrine, which (within the clinically relevant dose range of approximately 0.01 to $0.1 \mathrm{mcg} / \mathrm{kg} / \mathrm{min}$ ) supports the circulation predominantly through positive inotropic effects. Patients with normal LV function typically receive norepinephrine, which (at doses again titrated between approximately 0.01 and 0.1 $\mathrm{mcg} / \mathrm{kg} / \mathrm{min}$ ) supports the circulation predominantly through its vasoconstrictor effect. If either agent individually is inadequate to maintain the blood pressure, the other agent is typically added. Our experience, as here described, suggests that administration of either epinephrine, norepinephrine or both simultaneously via a continuous, low-dose infusion initiated prior to rapid pacing (rather than as boluses after pacing) allows for a more rapid hemodynamic recovery and intraoperative course.

Figure 3 illustrates this point. The ratio of systemic arterial blood pressure to pulmonary arterial blood pressure has been shown to reflect relative LV performance [4]. A higher ratio generally reflects improved performance. Here, in the "reactive management" case, this ratio was initially higher than in the other two cases, suggesting relatively stronger LV function. However, this patient's LV dilated significantly after balloon valvuloplasty (most likely due to the acute severe aortic regurgitation that this procedure caused), resulting in systemic hypotension and pulmonary arterial hypertension (Figure 3, reactive, post valvuloplasty). Hence, at the end of this case, the ratio of systemic systolic arterial blood pressure to pulmonary arterial systolic blood pressure was lower than it had been at the beginning. Additionally, as illustrated by Figure 3, both of the patients managed 


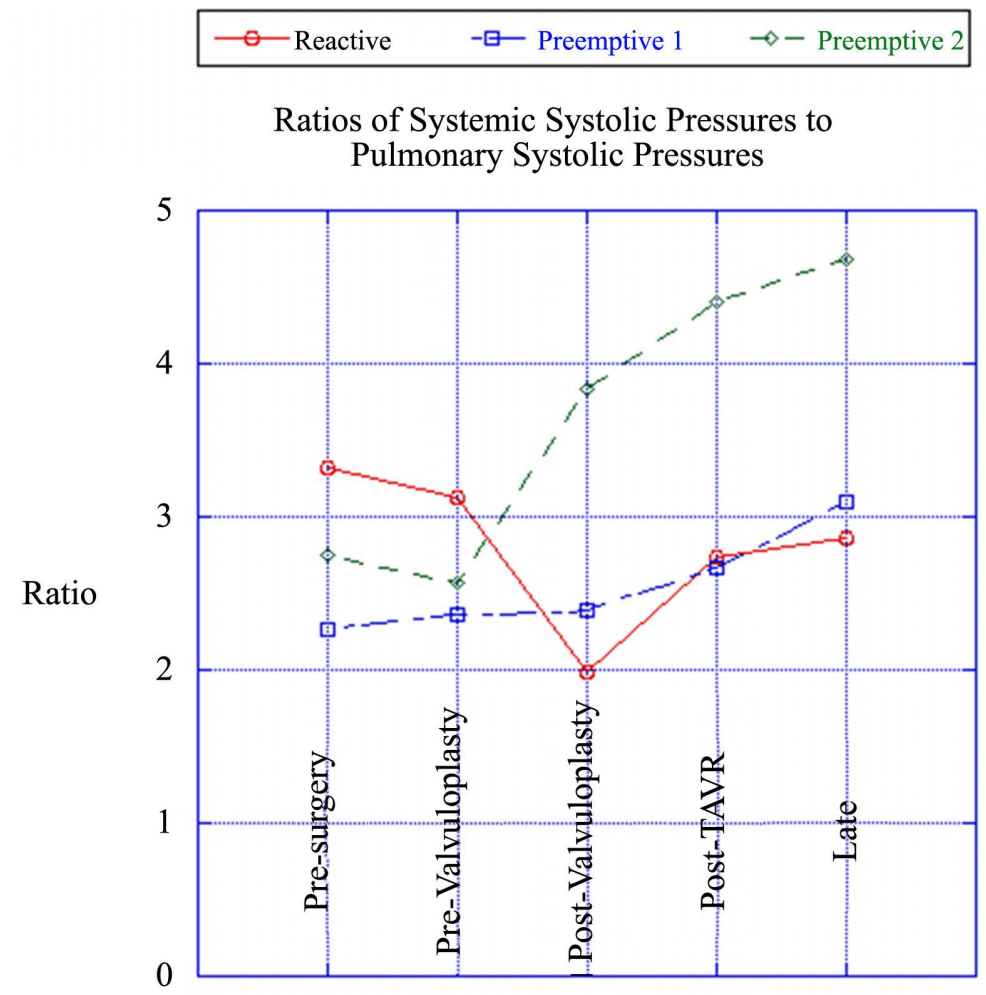

Figure 3. Changes in the ratios of systemic systolic pressures to pulmonary systolic pressures at different stages of TAVR.

with the preemptive strategy had relatively higher ratios at the end of the case, suggesting an association between this preemptive management strategy and relatively decreased impairment of LV function after TAVR. However, as this is a retrospective review of a small cohort of patients, the observed association does not establish causality. Hence additional research in this area is needed, ideally including prospective trials that are adequately powered to clearly delineate and establish optimal strategies for both selection and dosing of vasoactive agents during this important new procedure.

\section{References}

[1] Leon, M.B., Smith, C.R., Mack, M., Miller, D.C., Moses, J.W., Svensson, L.G., Tuzcu, E.M., Webb, J.G., Fontana, G.P., Makkar, R.R., Brown, D.L., Block, P.C., Guyton, R.A., Pichard, A.D., Bavaria, J.E., Herrmann, H.C., Douglas, P.S., Petersen, J.L., Akin, J.J., Anderson, W.N., Wang, D. and Pocock, S., for the PARTNER Trial Investigators (2010) Transcatheter Aortic-Valve Implantation for Aortic Stenosis in Patients Who Cannot Undergo Surgery. New England Journal of Medicine, 363, 1597-1607. http://dx.doi.org/10.1056/NEJMoa1008232

[2] Billings, F.T., Kodali, S.K. and Shanewise, J.S. (2009) Transcatheter Aortic Valve Implantation: Anesthetic Considerations. Anesthesia and Analgesia, 108, 1453-1462. http://dx.doi.org/10.1213/ane.0b013e31819b07ce

[3] Klein, A.A., Skubas, N.J. and Ender, J. (2014) Controversies and Complications in the Perioperative Management of Transcatheter Aortic Valve Replacement. Anesthesia and Analgesia, 119, 784-798. http://dx.doi.org/10.1213/ANE.0000000000000400

[4] Robitaille, A., Denault, A.Y., Couture, P., Belisle, S., Fortier, A., Guertin, M.C., Carrier, M. and Martineau, R. (2006) Importance of Relative Pulmonary Hypertension in Cardiac Surgery: The Mean Systemic-to-Pulmonary Artery Pressure Ratio. Journal of Cardiothoracic and Vascular Anesthesia, 20, 331-339. http://dx.doi.org/10.1053/j.jvca.2005.11.018 